Purdue University Purdue e-Pubs

$1-1-2013$

\title{
Usability evaluation of a research repository and collaboration website
}

Tao Zhang

Purdue University, zhan1022@purdue.edu

Deborah J.Maron

deborah.maron@gmail.com

Christopher C. Charles

Purdue University, cccharle@purdue.edu

Follow this and additional works at: http://docs.lib.purdue.edu/lib_fsdocs

Part of the Library and Information Science Commons

\section{Recommended Citation}

Tao Zhang, Deborah J. Maron \& Christopher C. Charles (2013) Usability Evaluation of a Research Repository and Collaboration Web Site, Journal of Web Librarianship, 7:1, 58-82, DOI: 10.1080/19322909.2013.739041

This document has been made available through Purdue e-Pubs, a service of the Purdue University Libraries. Please contact epubs@purdue.edu for additional information. 
Usability Evaluation of a Research Repository and Collaboration Website

Tao Zhang, zhan1022@purdue.edu

Deborah J. Maron, dmaron@purdue.edu

Christopher C. Charles, cccharle@ purdue.edu

Purdue University Libraries, 504 W. State Street, West Lafayette, IN 47907, USA 


\begin{abstract}
This paper reports results from an empirical usability evaluation of HABRI (HumanAnimal Bond Research Initiative) Central as part of the effort to develop an open access research repository and collaboration platform for human-animal bond researchers. By repurposing and altering key features of the original HUBzero system, HABRI Central hosts previously published materials from related disciplines and an extensive bibliography, in addition to traditional hub materials such as tools and datasets. Seven graduate students in the College of Veterinary Medicine at Purdue University participated in the usability evaluation. Tasks included exploring the system, finding an article in the repository, submitting an article to the repository, adding bibliographic information of an article to the repository, and using user groups and questions and answers. Participants also answered open questions regarding their overall experience and rated HABRI Central's usability using the System Usability Scale (SUS). Response measures included task successfulness, navigational steps, task time, participant comments and behavior notes recorded by the researcher. Results of the evaluation showed that the overall user experience of HABRI Central was satisfactory, but also indicated a number of usability issues. Participants had difficulty inputting metadata such as resource type and author information when submitting an article to the repository. Participant comments and researcher notes during the article submission process indicated that the workflow could be further improved to reduce user confusion. There were also interface design issues regarding layout and consistency. It is expected that findings from this study and the evaluation methodology can be extended to the development and evaluation of similar research repository systems.
\end{abstract}


Keywords: research repository, usability evaluation, human-animal bond, HUBzero, open access, research collaboration 


\section{Introduction}

Research repositories have been developed and implemented rapidly in research institutions for collecting, sharing, and preserving research materials and data electronically and in accordance with the goal of open access. Institutional repositories built based on digital repository systems like DSpace (Smith et al. 2003), EPrints (Gutteridge 2002), and Fedora (Staples, Wayland, and Payette 2003) have been emphasized as a key element of cyberinfrastructure for academic research libraries to manage and disseminate digital research materials and data (ARL Digital Repository Issues Task Force 2009; Lynch 2003). There has also been growing demand of developing discipline-centric (i.e., subject-specific) research repositories to promote discovery of new studies and expand the availability of research materials among researchers of similar interests.

Although the necessity and advantages of research repositories for scholarly communication have been widely accepted and issues of system implementation have been frequently discussed in the scientific community, there has been a lack of studies concentrating on the usability of research repositories for end users, particularly content contributors. Usability of research repositories may affect user motivation and engagement, as well as the way in which the research repository system is utilized. Because of the diversity of experience and information needs of potential users, it is a challenge for research repository systems to provide users with an efficient, effective, and satisfactory experience.

The present study is part of the effort to develop an online platform for research and collaboration into the relationship between humans and animals. The HABRI 
(Human-Animal Bond Research Initiative) Central website (http://habricentral.org) is designed to facilitate the study of human-animal bonds across disciplines ranging from medicine, nursing, public health, veterinary medicine, education, and beyond. By bringing together the resources of many disciplines under one location, researchers can develop a better understanding of the broad direction of the field while making use of specialized knowledge developed in previously unknown or inaccessible areas.

\section{Related Work}

\section{Usability of Research Repositories and Evaluation Methods}

In order to evaluate and improve the usability of research repositories, the definition of the usability concept as well as appropriate metrics and evaluation methods must be reviewed and determined for this particular context. Previous usability studies have examined digital libraries, which may shed light on usability evaluation of research repositories. In general, usability of digital libraries has been defined as how easily and effectively users can find information through their interaction with the digital library interface (Chowdhury, Landoni, and Gibb 2006).

Jeng $(2005 \mathrm{~b}, 2005 \mathrm{a})$ reviewed the definition of usability and applicable evaluation methods in the context of digital libraries. She proposed an evaluation model with metrics for assessing usability of academic research libraries. The model included four interrelated dimensions: effectiveness, efficiency, satisfaction, and learnability. Similarly, Buchanan and Salako (2009) developed an evaluation framework in which effectiveness, efficiency, aesthetic appearance, terminology, navigation, and learnability are key attributes of digital library usability. Tsakonas and Papatheodorou (2008) identified ease 
of use and learnability as important attributes of open access digital repositories' usability that can affect user preference and satisfaction. The research findings by Jeng, Buchanan and Salako, and Tsakonas and Papatheodorou further expanded the general definition suggested by Chowdhury, Landoni, and Gibb (2006). On a more detailed level, Hariri and Norouzi (2011) summarized evaluation criteria for digital libraries' user interfaces including feedback, ease of use, match between system and the real world, customization, user support, user workload, interaction, compatibility, visibility of system status, user experience, flexibility, and accessibility.

More importantly, as research repositories have extended digital libraries with self-archiving and publishing capabilities (i.e., allowing users to contribute to the repository), it is expected that the usability of research repositories will also measure the ease of contributing research materials to the repository. Feng and Huang (2008) used a set of evaluation criteria including effectiveness, efficiency, and satisfaction with subdimensions and corresponding metrics to evaluate usability of three popular discipline repositories (arXiv, PubMed Central, and E-LIS). As a result of their study, they state that the efficiency of a discipline repository should be measured in both archiving and searching processes.

A wide range of usability evaluation methods have been applied to the domain of digital libraries and repositories, including analytical methods like heuristic evaluation (Hartson, Shivakumar, and Pérez-Quinones 2004; Blandford et al. 2004); cognitive walkthrough (Blandford et al. 2004); empirical methods using direct involvement and observation of users such as questionnaire survey (Veiga e Silva, Gonçalves, and Laender 2007); usability tests (Kim and Kim 2008); task performance measures and interviews 
(Ferreira and Pithan 2005); and focus groups (Dobreva and Chowdhury 2010). Most usability evaluations in the literature were focused on the information retrieval process (i.e., how users interact with the system to find information), and a few studies discussed the usability of digital repository system installation and configuration (e.g., DSpace; Körber and Suleman 2008). As content contribution becomes an important part of research repository development, there will be an increasing need to evaluate the usability of the submission process for research repositories.

\section{Usability Evaluations of Information Seeking in Research Repositories}

A large number of previous studies on usability of research repositories focused on the interaction between the information seeker and content in the repository. Specifically, those studies evaluated how the interface of research repositories assists users to find and retrieve information.

Kim (2005) conducted a heuristic evaluation and usability test of the search interface of two commonly used repository systems (DSpace and EPrints) at the Australian National University. The usability test involved 18 undergraduate students in a between-subjects experiment design. Participants were asked to complete nine searching tasks involving simple and advanced searches and one browsing task with the two systems. Task performance measures included time for completing tasks, number of errors, and after-test satisfaction ratings. The results showed that participants using DSpace had shorter task time, fewer errors, and higher satisfaction than participants using EPrints. 
Xie (2008) recruited 19 participants to evaluate two digital libraries (American Memory and University of Wisconsin Digital Collections). Usability was part of the evaluation scheme. Participants kept a diary and recorded search time, queries, browse paths, use of help, and problems encountered. The recorded data showed patterns of digital library use and identified system features affecting participants' usability ratings, including: (1) search and browse functions, navigation points, and view and output options (e.g., adjustable font size); (2) customized interface for collections; and (3) contextualized help.

McKay and Burriss (2008) reported a case study of using heuristic evaluation to test and improve the usability of an institutional repository system (Swinburne Research Bank at Swinburne University of Technology, Australia). They conducted background research on information-seeking behavior and information systems, empirical surveys on user understanding of terminology, and comparison of a similar search interface, in order to inform and guide the heuristic evaluation. The heuristic evaluation led to improved interface navigation and search results display for information seekers.

Kim and Kim (2008) conducted in-lab and remote usability tests with 30 participants, as well as a focus group interview with four experts on an institutional repository system ("dCollection") in Korea. They established a usability evaluation framework covering satisfaction, supportiveness, usefulness, and effectiveness. Based on the usability tests and interview, they provided suggestions for improving visual appearance of user interface, metadata submission, and search and browse functions. 


\section{Usability Evaluations of Content Contribution in Research Repositories}

Veiga e Silva, Gonçalves, \& Laender (2007) reported an evaluation of selfarchiving service for the Brazilian Digital Library of Computing (BDB-Comp) involving potential user groups including archiving experts, computer science professors, and graduate students. In the evaluation, subjects performed a set of tasks to submit three types of work to the BDB-Comp, completing an evaluative questionnaire at the end of the tasks. The questionnaire measured subjects' previous experience of and knowledge about self-archiving and metadata, as well as ease of use, comfort, and usefulness of the BDBComp self-archiving service. The results showed that participants found the service easy to learn and useful, and experts tended to perform tasks better and give higher ratings in the questionnaire than the other two user groups.

Caccialupi, Calvi, Cassella, and Conte (2009) reported a usability evaluation of a multimedia repository interface based on DSpace 1.5. In addition to confusions caused by terminology, usability issues of content submission and workflow were identified from the evaluation, including problems recognizing the upload link, unclear compulsory fields for metadata, and fragmented and redundant workflow. They also proposed design guidelines for improving the usability of the DSpace system. Boock (2005) conducted a quick and simple usability test of the submission process of the DSpace electronic theses and dissertation collection at Oregon State University Libraries, recruiting six undergraduate students and asking them to submit a thesis to the collection. The usability test results revealed potential usability issues of email authentication and user instructions.

Other studies discussed factors affecting researcher participation in institutional repositories and approaches to addressing researchers' motivations. Davis and Connolly 
(2007) identified a number of reasons why faculty members did not use institutional repositories, including redundancy with other modes of scholarly communication, learning curve, copyright concerns, plagiarism, and whether posting a manuscript constitutes "publishing." From a survey of 31 faculty members who have research work deposited in an institutional repository, Kim (2007) found a positive link between willingness to contribute to the repository and acceptance of open access concept. However, faculty members required by funding agencies to self-archive in repositories were much less likely than others to make further contributions to the institutional repository. Mark and Shearer (2006) reviewed content recruitment strategies for populating institutional repositories, including general promotional activities, depositing services (non-author depositing and copyright assistance), content harvesting, building researcher bibliographies, providing usage and citation information, and implementing university policies.

There is a lack of understanding on how the content submission process itself may have hindered self-archiving practices. Swan and Brown (2005) found that the perceived time requirement and possible technical difficulties in depositing research work may cause the reluctance of researchers to self-archive. Xia and Sun (2007) examined selfarchiving practices in nine well-known open access institutional repositories using the EPrints system. They found that the majority of deposits in the repositories were contributed by non-authors; that is, the practice of self-archiving for institutional repositories had not been widely accepted by researchers. They also found that the availability of full text in those repositories was relatively low, Australian repositories being an exception. Xia and Sun suggested that one possible reason of the low rate of 
author self-archiving was the use of a liaison system where librarians working with departments deposited works of faculty members.

\section{The HABRI Central Research Repository}

The HABRI Central website serves as an open access research repository for human-animal bond research materials (literature, data sets, videos, etc.) from all relevant disciplines. HABRI Central promotes new research by facilitating every stage of the research cycle, including enhancing discoverability of existing research, facilitating collaborations between scholars, increasing visibility of new research, and providing a stable storage space for all research content. Specifically, through this centralized research repository across disciplines, researchers will be able to discover research materials on human-animal bond that may fall outside their normal scope of attention. Researchers can freely access a wide array of published texts through the repository and bibliography on HABRI Central. More importantly, they can submit materials and publications to the repository and share their research work with the public. HABRI Central also allows users to form and join groups of similar interests, which facilitates discovering and connecting with experts, professionals, and others involved in the study of human-animal interaction. Another community resource, Questions and Answers, allows users to vote for the best answers of responses provided by users. Users can also write wiki-type articles on a particular topic (monitored by the HABRI Central team) as part of the knowledge base curated on HABRI Central. The repository and community resources available on HABRI Central are shown in [PLACE FIGURE 1. 


\section{[PLACE FIGURE 1 HERE]}

HABRI Central is built upon the HUBzero platform for collaborative scientific research (McLennan and Kennell 2010) which is itself built upon the Joomla! content management system (www.joomla.org). The HUBzero platform was selected as the foundation of the HABRI Central project for its strong track record of facilitating knowledge exchange around content repositories. Originally designed as a way to host and share simulation tools among the nanotechnology community, HUBzero has since grown into a versatile repository and content management system suitable for different content types. Many of the aforementioned features were already available in partial or full form as part of the HUBzero system. Only minor changes were needed to accommodate HABRI Central as proposed.

As mentioned, HABRI Central plays host to a wide array of materials, including audio, book chapters, books, conference papers, conference proceedings, datasets, government documents, journal articles, magazine articles, newspaper articles, pamphlets, posters, presentations, reports, soft literature or narratives, still images, theses, tools, and videos. A HABRI Central-specific metadata schema was devised to accommodate this diversity, which consists of an amalgam of basic bibliographic or descriptive fields and Dublin Core fields, some of which map to each other. The schema mapping was laid out in a spreadsheet matrix that compared basic bibliographic fields in one column against Dublin Core fields in the next column; for instance, author in the bibliographic field column mapped to creator in the Dublin Core column, and ISBN in the bibliographic column mapped to identifier in Dublin Core. Once the spreadsheet schema was complete, the metadata fields were entered into HUBzero using human-readable names. 
There are several reasons Dublin Core was chosen to be part of the HABRI Central metadata schema. Firstly, it was chosen because of its simplicity, as non-librarian end users (e.g., lay people, veterinarians, researchers, faculty and students) will be the ones submitting content to HABRI Central. Further, it was decided that certain Dublin Core fields' values such as dc:format (MIME type) could be automatically saved in the back end without end-user intervention, thereby minimizing the chance of users inputting illegal values in selected fields. Finally, a librarian and development team at Purdue University Libraries provided a Joomla! component that turns HUBzero into an OAIPMH data provider; that is, data from selected fields are mapped and serialized to DCXML, and this data can then be ingested into discoverability tools such as Ex Libris Primo.

\section{Motivation}

Prior to HABRI Central's launch in March of 2012, an initial user survey was conducted to better understand the expectations and usage habits of HABRI Central's likely users. The survey contained 34 questions, including logic-contingent probes (i.e., "if... then..." questions), on subjects including basic demographics, Internet usage habits, information searching habits, virtual community involvement, and users' involvement with the human-animal bond. The survey was distributed among project collaborators and their colleagues. Out of ten respondents, six completed the survey. The information gathered from this survey reinforced prior assumptions about potential HABRI Central users, including their familiarity with online academic databases and mixed affiliation with academic institutions. The survey responses also suggested ways to enhance the 
value of HABRI Central's library content, including indicating whether or not a resource is peer-reviewed and including abstract and other supplementary information where possible.

While the results of the survey suggested that we had the right types of people in mind when developing HABRI Central, they told us nothing of how easily users could engage with the platform. Previous studies have defined usability in digital libraries and repositories but the concept was mostly limited to the experience of information seeking. As user input becomes critical to the growth of research repositories, there is a need to empirically evaluate HABRI Central as a research repository from both information seeker and resource contributor perspectives. It was expected that the evaluation results would lead to design implications for improving the usability of HABRI Central and similar systems based on the HUBzero platform. Furthermore, the usability evaluation methodology applied in this study involving potential users could be extended to other research repositories to reveal issues of interface design and user preferences affecting critical task performances.

\section{Method}

\section{Participants}

Seven participants ( 5 female and 2 male) were recruited for the usability evaluation. Five of them were graduate students in the College of Veterinary Medicine at Purdue University; one female participant had a master degree in library science; and another female participant had a master degree in social science. Participants had generally extensive experience of finding books and articles on library websites and scholarly 
databases (see [PLACE TABLE 1). None of the participants had experience of using institutional or research repositories. Participants ranged in age from 26 to $46(M=33.3$, $S D=7.4)$

\section{[PLACE TABLE 1 HERE]}

\section{The HABRI Central Interface}

The main page of HABRI Central consists of several functional areas as shown in : (1) a drop down navigation menu; (2) featured resources, articles, and discussions; (3) Upcoming Events; (4) Latest Resources; (5) Latest Discussions; and (6) Latest Questions. The navigation menu includes Library: Bibliography, Repository, Tags; Community: Members, Contributors, Groups, Forum, Questions \& Answers, Events, Topics, Jobs, Feedback; About: About HABRI Central, Sponsors. Users can access help options and documentation by clicking on the "Need Help?" link on the top right corner of every page. Links to user login and registration and a system-wide search are at the right of the navigation menu and above the features area.

\section{[PLACE FIGURE 2 HERE]}

Users can access resources in the repository through either the navigation menu's Library-Repository option or the Latest Resources area. [PLACE FIGURE 2 shows the Resources interface. Resources are user-submitted pieces of content that can include video presentations, publications, or simulation tools. Users can contribute their research materials to HABRI Central by clicking on the "Submit a resource" button and going through a guided process. Similarly, users can access the bibliography through the navigation menu (Library-Bibliography) and they can also submit bibliographic information through the Citations interface (See [PLACE FIGURE 3). Both resources 
and citations are part of the research materials and information about research materials stored in HABRI Central. The difference between resources and citations is that citations only include bibliographic information whereas resources have actual files stored in HABRI Central. Users can directly download resources from HABRI Central; for citations, HABRI Central provides link resolver links for users to locate full text and other scholarly services (e.g., interlibrary loan). Two of the community resources of HABRI Central, Groups and Questions and Answers, are shown in Figures 5 and 6.

\section{[PLACE FIGURE 2 HERE]}

[PLACE FIGURE 3 HERE]

[PLACE FIGURE 4 HERE]

\section{[PLACE FIGURE 5 HERE]}

\section{Tasks}

Tasks participants performed in the usability evaluation included: (1) finding an article in the repository; (2) submitting an article to the repository; (3) adding a citation to an article to the HABRI bibliography; and (4) asking a question in the Questions and Answers area and joining a Group and posting a message in that group's discussion area. For the "finding an article" task, participants were given the citation information of a journal article and asked to locate that article in the repository and in the bibliography. Participants were provided both citation information and an actual file for the article submission task. They were asked to input the correct metadata (article type, title, author, year of publication, etc.) from the citation information and upload the file into the repository. They also needed to select a copyright license agreement for the article as part 
of the submission process. Participants were asked to input only metadata for a journal article for the adding a citation task.

\section{Response Measures}

Response measures of the usability evaluation included: (1) successfulness of each task; (2) whether participants needed help from the researcher during each task; (3) number of steps of the navigational path participants went through in each task; (4) time to complete each task; (5) participants' comments during each task; and (6) participants' usability ratings of HABRI Central, measured by the System Usability Scale (SUS; Brooke 1996). The researcher also took notes about participants' behavior during the tasks, such as pauses in continuous mouse movements, facial expressions, and body language.

\section{Procedure}

Upon arrival, participants were first briefed about the purpose of the usability evaluation. They read and signed a consent form and completed a simple demographic survey regarding their experiences of scholarly search and databases. At the beginning of the evaluation, participants explored HABRI Central and reported things they noticed on the website. They checked the menus and reported any items they had difficulty of understanding. They viewed major sections of the homepage (Upcoming Events, Latest Resources, Latest Discussions, and Latest Questions) and commented on the purposes of those sections based on their perception. Participants were also asked to locate the area where they could seek help for using HABRI Central. After the initial exploration, participants completed the tasks in the order given above. They were encouraged to talk aloud about their expectations, difficulties, and general comments about HABRI Central 
during the tasks. Participant activities on the computer screen and voice were recorded using Morae software (TechSmith 2012). A researcher sat next to participants, answered questions from participants during the tasks, and made observation notes about participant behavior. The researcher provided participants with necessary assistance only when participants explicitly requested and they were unable to proceed. After the tasks, participants answered open questions and completed the SUS questionnaire about their overall experience of HABRI Central. Each evaluation session lasted approximately one hour.

\section{Results}

\section{Descriptive Statistics}

A majority of the participants completed the testing tasks with no assistance from the researcher. The descriptive statistics for response measures of each testing task (except for task successfulness) across participants are shown in [PLACE TABLE 2. Participant responses to the SUS questionnaire regarding their overall experience of HABRI Central are summarized in [PLACE TABLE 3. The range of rating for each statement of the SUS questionnaire is 1 (strongly disagree) to 5 (strongly agree). Participant ratings for Statements $2,4,6,8$, and 10 were reversed (e.g., changing original rating of 1 to 5 to make the direction of rating consistent with other statements) to calculate the total SUS score (see Table 4; higher score means higher usability rating). 
[PLACE TABLE 2 HERE]

[PLACE TABLE 3 HERE]

[PLACE TABLE 4 HERE]

\section{Usability Issues and Suggestions}

Through observation of participants performing tasks, we identified a few potential usability issues related to HABRI Central's article submission workflow, user input design, and positions of certain interface elements. The workflow of submitting research materials to HABRI Central showed that resources (essentially metadata plus the actual file of the journal article, book, book chapter, video, etc.) and citations (just metadata) are managed separately through the repository and bibliography, respectively. Participants seemed to have difficulty understanding repository and bibliography as two separate spaces on HABRI Central. For example, Participant 6 commented: "It is a little confusing, the bibliography versus repository. It seems that they are looking like basically the same thing." When submitting a journal article to HABRI Central, participants had to go through two separate processes for uploading to the repository and bibliography (see [PLACE FIGURE 6) and input the same metadata (e.g., title, author, year of publication, DOI, etc.) for each process. HABRI Central provides a way of linking citations in the bibliography to resources in the repository, but it is redundant, and none of the participants understood the interface for creating the links and were able to correctly use it.

\section{[PLACE FIGURE 6 HERE]}

Another usability issue was the interface design for adding authors as part of the resource submission process ([PLACE FIGURE 7). The interface was designed so that as 
the user types the author name in the author name text box, the system will try to search the name in existing contributors of HABRI Central and allow the user to select the matched author from a dropdown list. However, most participants did not know only one author name could be entered at a time in order for the searching and matching function to work. They system always returned a zero match after participants typed two or more names in the author name text box. After clicking on the "Add" button to add the author in the text box to authors list shown in the table view, the system required the user to click on the "Save Changes" button before moving on to the next step. Most of the participants thought the authors were already added to the authors list after they clicked on the "Add" button, so they did not click on the "Save Changes" button and the author information they inputted was lost in the remaining steps of the process. A few participants did not notice the author they typed in was added to the table view below, because the system did not give any other feedback after they clicked on the "Add" button. Because of all these issues, participants spent longer time in this step, making the total time for completing the article submission task significantly longer.

\section{[PLACE FIGURE 7 HERE]}

A number of usability issues were related to the layout of interface elements, which resulted in longer time than normally required for participants to understand how the interface worked or where to start. For example, the first step of the article submission task was to select the resource type (audio, book sections, books, conference papers, journal articles, etc.). The resource type selection was placed on the very left part of the interface as a side column ([PLACE FIGURE 8), which made participants think it was not a primary piece of information. Participants spent a significant amount of time 
trying to find out where to start and go to the next step on this page. Another example was that the "New Question" link was placed on the top right corner of the Questions and Answers interface, which is not a usual position for participants to look when they were asked to submit a new question.

[PLACE FIGURE 8 HERE]

\section{Discussion}

In general, participants' task performance showed that searching for materials and using the community features of HABRI Central was easy and without major obstacles. Most participants completed these two tasks within reasonable time duration (averaging about two minutes) and did not require extensive assistance from the researcher. However, participants encountered difficulties and confusion during the article and citation submission tasks, as the performance data and our observation showed. It took at least seven minutes to submit an article, with an average over 10 minutes. An important reason for this difference is the usability issues of user input and interface layout at steps of the processes including selecting resource type and adding authors of the resource. The intention of the interface design was not communicated well to participants, and they were unable to relate their knowledge of common websites to the HABRI Central interface. In addition, HABRI Central did not provide enough guidance and help for participants to learn the system through interaction. All of these issues could have considerably increased the time participants needed to understand the interface based on initial interactions. 
Another important source of participant confusion was the way resources and bibliographic information (i.e., citations) were presented separately by HABRI Central's repository and bibliography. Because HABRI Central was developed based on the HUBzero platform, its infrastructure was not explicitly designed to meet the requirements of research repositories. From the user's perspective, a research repository should have a single storage space for all types of information including citations without actual files associated and stored in the repository. For system implementation reasons, the version of HABRI Central evaluated in this study had to use the repository to store resources with actual files and bibliography to store citations that are linked to external site for accessing actual files of full text. The discrepancy between user expectations and system design contributed to the confusions expressed by participants. To remedy this discrepancy, we have proposed a new task flow based on participant expectation of HABRI Central as one storage space for all information ([PLACE FIGURE 9). Conceptually, this new workflow handles all types of materials with consistent steps and the user has to input resource metadata only once. Implementing this workflow requires changes to be made to HABRI Central's system architecture, but this new workflow will improve the overall user experience by simplifying the tasks for users and thus attracting more contributions from users.

\section{[PLACE FIGURE 9 HERE]}

We have proposed new designs to address the usability issues from the evaluation. For adding authors during resource submission process, we suggest enhancing the search functionality associated with the author name text box so that the search will not be restricted to only one name. The response speed of the search should be improved so that 
as the user types in the author name textbox, the system will constantly search and suggest matched existing HABRI Central contributors in the dropdown list (see [PLACE FIGURE 10). The author list will automatically save any changes so the user no longer has to manually save them. We will also add descriptions and tool tips to explain how the author name text box works and what the author list is. Another proposed change includes moving resource type selection to the center of the page and adding visual cues to show it is the first step of resource submission. Finally, important action links such as "New Question" should be moved to the left and above the questions list.

\section{[PLACE FIGURE 10 HERE]}

Despite the usability issues revealed from the evaluation, participants valued the utility of HABRI Central for human-animal bond research materials and rated highly their overall experience of HABRI Central. Most participants liked the idea of using a dedicated research repository to curate all materials related to broad topics across disciplines such as human-animal bond. They also commented that they would share their research materials and publications on HABRI Central, even though the submission process could be further improved. Participants agreed that the open access nature of HABRI Central and the community features could lower the boundaries between disciplines and facilitate collaborations among researchers.

In order to design and implement research repositories similar to HABRI Central and ensure user performance and ease of use in critical tasks, there is a need for a structured design approach driven by user experience requirements. Based on our experience of developing HABRI Central and the usability evaluation results, we have identified a user-centered design process for developing and evaluating research 
repositories as shown in [PLACE FIGURE 11. The first stage of the process is to identify potential users, their expectations of the role of a research repository in their work routine, and how their research could benefit from incorporating a research repository. Findings of user research would then be analyzed to form specifications and requirements to drive the design of tasks the system will need to support, the processes that users need to go through to complete those tasks, and the overall interaction between users and the system. The outputs of the design stage include documents like wireframes showing interface layout and use cases showing workflows, which are the basis for the system implementation stage. For system implementation, a research repository and collaboration platform needs to be adopted or developed to meet a number of requirements including features and functionalities, cost of implementation, technical support, and software and hardware requirements. An important part of the implementation would be building a metadata system for resources stored in the repository. The metadata schema needs to contain fields necessary for discoverability in a simple search within the repository, as well as discoverability via protocols like OAIPMH. In the evaluation stage, participants from the potential user population should be involved. The evaluation should collect measures of task performance, participant comments, and behavior notes made by experienced usability researchers. Usability issues with supporting evidence identified from the evaluation should be fed back to the design stage for iterative refinement and improvement. Suggestions of how the interface or interaction could be modified should be aimed at addressing those usability issues while minimizing the effect on system architecture and development efforts. 


\section{[PLACE FIGURE 11 HERE]}

\section{Conclusion}

The objective of this study was to evaluate the usability of HABRI Central as a research repository and collaboration platform for human-animal bond researchers. Participants from potential users were recruited for the evaluation and they performed both information seeking and content contribution tasks. The evaluation collected task performance measures, participant comments, and observational notes in order to identify workflow and usability issues. The results revealed that the separation of resources and bibliography due to adaptation of the HUBzero platform caused some confusion among participants, and certain steps of the resource submission process (e.g., selecting resource type and adding authors) could be improved to provide clear guidance and facilitate user input. The usability evaluation also showed that the HABRI Central interface should conform to common web layout guidelines so that users can relate past experiences to using functional links on HABRI Central.

One limitation of the evaluation was the limited number of participants, which could not lead to statistically significant results. However, the evaluation was part of an iterative design process and its objective was to identify usability issues and provide design suggestions through task performance measures and subjective feedback. Because we conducted an initial user survey to identify potential users of HABRI Central, the participants recruited for the usability evaluation were highly representative of potential user groups. We also noticed that as we tested HABRI Central with more participants, we observed similar behavior patterns and usability issues with new participants, which is common in usability research practice (Nielsen and Landauer 1993). 
As users are accepting research repositories as importance resources and tools in their work, it is critical to define and evaluate system usability in all tasks including information seeking and browsing, content contribution and publishing, and collaborative features. The evaluation methodology in this study involving potential users and task performance measures can be integrated to a structured user-centered design process for improving HABRI Central in the future and building similar research repositories systems.

\section{Acknowledgements}

We thank Charles Watkinson and Dr. Alan Beck for their support of this study. We also thank HUBzero team for their development work on HABRI Central.

\section{References}

ARL Digital Repository Issues Task Force. 2009. The Research Library's Role in Digital Repository Services. Washington, DC: Association of Research Libraries.

Blandford, Ann, Suzette Keith, Iain Connell, and Helen Edwards. 2004. "Analytical usability evaluation for digital libraries: A case study." Proceedings of the 2004 Joint ACM/IEEE Conference on Digital Libraries:27-36.

Boock, Michael. 2005. Improving DSpace@OSU With a Usability Study of the ET/D Submission Process. Ariadne (45), http://www.ariadne.ac.uk/issue45/boock/.

Brooke, John. 1996. "SUS-A quick and dirty usability scale." In Usability Evaluation in Industry, edited by P. W. Jordan, B. Thomas, B. A. Weerdmeester and A. L. McClelland. London: Taylor and Francis. 
Buchanan, Steven, and Adeola Salako. 2009. "Evaluating the usability and usefulness of a digital library." Library Review no. 58 (9):638-651.

Caccialupi, Roberta, Licia Calvi, Maria Cassella, and Georgia Conte. 2009. "Usability Evaluation of a Multimedia Archive: B @ bele." Proceedings of the 13th European Conference on Digital Libraries, LNCS 5714:370-376.

Chowdhury, Sudatta, Monica Landoni, and Forbes Gibb. 2006. "Usability and impact of digital libraries: A review." Online Information Review no. 30 (6):656 - 680.

Davis, Philip M., and Matthew J.L. Connolly. 2007. "Institutional repositories: Evaluating the reasons for non-use of Cornell University's installation of DSpace." D-Lib Magazine no. 13 (3/4).

Dobreva, Milena, and Sudatta Chowdhury. 2010. "A User-Centric Evaluation of the Europeana Digital Library." In The Role of Digital Libraries in a Time of Global Change, edited by Gobinda Chowdhury, Chris Koo and Jane Hunter, 148-157. Springer Berlin / Heidelberg.

Feng, Qing, and Ruhua Huang. 2008. "Evaluating the Usability of Discipline Repositories." Proceedings of 2008 IEEE International Symposium on IT in Medicine and Education:385-390.

Ferreira, Sueli Mara, and Denise Nunes Pithan. 2005. "Usability of digital libraries: A study based on the areas of information science and human-computerinteraction." OCLC Systems \& Services no. 21 (4):311-323.

Gutteridge, Christopher. 2002. GNU EPrints 2 Overview. In 11th Panhellenic Academic Libraries Conference. 
Hariri, Nadjla, and Yaghoub Norouzi. 2011. "Determining evaluation criteria for digital libraries' user interface: a review." The Electronic Library no. 29 (5):698-722.

Hartson, H. Rex, Priya Shivakumar, and Manuel A. Pérez-Quinones. 2004. "Usability inspection of digital libraries: a case study." International Journal on Digital Libraries no. 4 (2):108-123.

Jeng, Judy. 2005a. "Usability assessment of academic digital libraries: Effectiveness, efficiency, satisfaction, and learnability." Assessment no. 55:96-121.

Jeng, Judy. 2005b. "What is usability in the context of the digital library and how can it be measured?" Information Technology and Libraries no. 24 (2):46-56.

Kim, Hyun Hee, and Yong Ho Kim. 2008. "Usability study of digital institutional repositories." The Electronic Library no. 26 (6):863-881.

Kim, Jihyun. 2005. "Finding documents in a digital institutional repository: DSpace and EPrints." Proceedings of the American Society for Information Science and Technology no. 42 (1).

Kim, Jihyun. 2007. "Motivating and impeding factors affecting faculty contribution to institutional repositories." Journal of Digital Information no. 8 (2).

Körber, Nils, and Hussein Suleman. 2008. "Usability of Digital Repository Software: A Study of DSpace Installation and Configuration." In Lecture Notes in Computer Science: Digital Libraries: Universal and Ubiquitous Access to Information., edited by George Buchanan, Masood Masoodian and Sally Cunningham, 31-40. Springer Berlin / Heidelberg.

Lynch, Clifford A. 2003. "Institutional repositories: Essential infrastructure for scholarship in the digital age." ARL: A Bimonthly Report (226). 
Mark, Timothy, and Kathleen Shearer. 2006. Institutional Repositories: A Review of Content Recruitment Strategies. In World Library and Information Congress: 72nd IFLA General Conference and Council. Seoul, Korea.

McKay, Dana, and Shaun Burriss. 2008. "Improving the usability of novel web software: an industrial case study of an institutional repository." Lecture Notes in Computer Science: Proceedings of the Web Information Systems Engineering no. 5176:102111.

McLennan, Michael, and Rick Kennell. 2010. "HUBzero: A Platform for Dissemination and Collaboration in Computational Science and Engineering." Computing in Science and Engineering no. 12 (2):48-52.

Nielsen, Jakob, and Thomas K. Landauer. 1993. "A mathematical model of the finding of usability problems." Proceedings of ACM INTERCHI'93 Conference:206-213.

Smith, MacKenzie, Mary Barton, Mick Bass, Margret Branschofsky, Greg McClellan, Dave Stuve, Robert Tansley, and Julie Harford Walker. 2003. "DSpace: An open source dynamic digital repository." D-Lib Magazine no. 9 (1).

Staples, Thornton, Ross Wayland, and Sandra Payette. 2003. "The Fedora Project: An open-source digital object repository management system." D-Lib Magazine no. 9 (4).

Swan, Alma, and Sheridan Brown. 2005. Open access self-archiving: An author study. [Departmental Technical Report].

Morae, http://www.techsmith.com/morae.html. 
Tsakonas, Giannis, and Christos Papatheodorou. 2008. "Exploring usefulness and usability in the evaluation of open access digital libraries." Information Processing \& Management no. 44 (3):1234-1250.

Veiga e Silva, Lena, Marcos André Gonçalves, and Alberto H.F. Laender. 2007. "Evaluating a digital library self-archiving service: The BDBComp user case study." Information Processing \& Management no. 43 (4):1103-1120.

Xia, Jingfeng, and Li Sun. 2007. "Assessment of self-archiving in institutional repositories: depositorship and full-text availability." Serials Review no. 33 (1):14-21.

Xie, Hong Iris. 2008. "Users' evaluation of digital libraries (DLs): Their uses, their criteria, and their assessment." Information Processing \& Management no. 44 (3):1346-1373. 